\title{
Evaluación y comparación de sistemas de alarma temprana en el perioperatorio del Hospital Ángeles Pedregal
}

\author{
Evaluation and comparison of early warning systems in the \\ perioperative period at Hospital Ángeles Pedregal
}

\author{
José Fernando Hernández Amador,* Ernesto Rojas Jiménez, ${ }^{\ddagger}$ Federico Armando Castillo González,\$ \\ Adriana Cecilia Martínez López, " María Angélica Maldonado Vázquez,\$ \\ Tania Karina Cázares Robles, * Mario Alberto Arredondo Ortiz*
}

Citar como: Hernández AJF, Rojas JE, Castillo GFA, Martínez LAC, Maldonado VMA, Cázares RTK et al. Evaluación y comparación de sistemas de alarma temprana en el perioperatorio del Hospital Ángeles Pedregal. Acta Med. 2021; 19 (1): 25-34. https://dx.doi.org/10.35366/98566

\section{Resumen}

Introducción: Durante el perioperatorio se presentan complicaciones que demoran más de lo esperado en identificarse y en ser corregidas debido al uso exclusivo del juicio clínico; el algoritmo de un EWS detecta oportunamente y abrevia el ingreso a la unidad de cuidados especiales. Existen diferentes algoritmos, nosotros comparamos el algoritmo que actualmente se usa frente a un MEWS conocido y validado. Objetivo: Comparar dos algoritmos de EWS en el perioperatorio del Hospital Ángeles Pedregal. Material y métodos: Durante dos meses contiguos se aplicó y comparó el rendimiento del MEWSHAP y MEWS en pacientes postoperados de cirugía general/gastrocirugía. Resultados: De 295 pacientes, hubo 21 complicaciones, seis ingresos a cuidados especiales, tres voceos que mostraron una sensibilidad de 66 (MEWSHAP) y de 33\% (MEWS) respectivamente. El grupo en general mostró tendencia a mayores complicaciones, proporcional a la edad y no se encontró relación con el tiempo quirúrgico. Conclusiones: Consideramos que el algoritmo actualmente vigente en nuestro hospital es apto para la aplicación en los pacientes quirúrgicos, aunque su aplicación no reemplaza la revisión clínica en cuanto a complicaciones tempranas que ameritan reoperación, pero abre la puerta para ajustes específicos en nuestra población.

Palabras clave: Deterioro clínico, algoritmos de EWS, clínico, escala de NEWS, equipo de respuesta rápida.

\section{Abstract}

Introduction: In the perioperative setting, complications take longer than expected to be identified and corrected due to the exclusive use of clinical judgment. The EWS algorithm promptly detects and shortens time of admissions to an Intensive Care Unit. Although several early warning algorithms exist, we compared the currently used model with a well-known and validated MEWS score. Objective: To compare two Early Warning Score algorithms in the perioperative setting at the Hospital Ángeles Pedregal. Material and methods: During a 60-day period, the currently used early warning system was compared to a validated EWS in patients that underwent general/gastrointestinal surgery. Results: A total of 295 patients were included. A total of 21 complications were detected, there were six admittance to ICU. Calls to the rapid response team were made three times, with a sensitivity of 66 and $33 \%$ for MEWSHAP and MEWS respectively. Complications in both groups were proportional to patients' age, and no relation was found with operative time. Conclusions: The currently used algorithm in our hospital is apt for use in surgical patients. While its application does not replace clinical assessment of complications that require reoperation, it opens the door to specific adjustments for our patient population.

Keywords: Clinical deterioration, EWS algorithms, clinical, NEWS scale, rapid response team.
* Médico Residente en Cirugía General, Facultad Mexicana de Medicina de la Universidad La Salle México, Hospital Ángeles Pedregal. Ciudad de México, México.

₹ Candidato a Doctor, Unidad de Biomedicina, Facultad de Estudios Superiores Iztacala, UNAM. México.

$\S$ Cirujano General, Cirujano Bariatra, Hospital Ángeles Pedregal. Ciudad de México, México.

ॠ Médico Cirujano, Universidad Popular Autónoma del Estado de Puebla. Puebla, México.

\section{Correspondencia:}

José Fernando Hernández Amador

Correo electrónico: cirugiaavanzadaybariatria@gmail.com

Aceptado: 01-04-2020.

www.medigraphic.com/actamedica 


\section{INTRODUCCIÓN}

Las admisiones no planeadas a terapia intensiva pueden provenir de los pisos de hospitalización posterior a cirugía programada, anexos de paraclínicos o urgencias y son por lo regular $0.7-1 \%$ de todas las admisiones. ${ }^{1}$ Se ha demostrado previamente que la morbilidad o mortalidad durante la estancia intrahospitalaria de un paciente no se presenta en general como una única alteración de manera abrupta, por lo que el uso único del juicio clínico del médico a cargo puede reconocer de manera tardía el deterioro clínico. La presencia de los mecanismos que llevan a este resultado en realidad se manifiesta con múltiples, y algunas veces ligeras, alteraciones en los signos vitales junto con ciertos datos clínicos que anteceden hasta por 24 horas la aparición de una complicación grave o la muerte, dichos elementos son tomados como signos de alarma. ${ }^{2}$

Morgan trabajó en 1997 el concepto de sistemas de alarma temprana (EWS, por sus siglas en inglés), los cuales fueron aplicados en pacientes quirúrgicos por primera vez por Stenhouse; ${ }^{3}$ se han implementado con diversos parámetros y escalas de manera local, y se han descrito más de 100 diferentes tipos, ${ }^{4-6}$ siendo unificados de manera nacional únicamente en Gran Bretaña, donde desde 2007 forman parte de las guías clínicas NICE. ${ }^{7}$ Se han obtenido experiencias y se han validado algunos puntajes en particular, pero sus objetivos terminales siempre son prevenir el retraso en la intervención o su oportuna transferencia al área de cuidados adecuados. Gardner Thorpe encontró una sensibilidad de $75 \%$ y una especificidad de $83 \%$ en pacientes que ameritaban ingreso a terapia intermedia o intensiva ${ }^{3}$ comparadas con una sensibilidad de $61 \%$ y una especificidad de $91 \%$ en el juicio clínico. ${ }^{3}$ En años recientes, la importancia de los conceptos de equipos de respuesta rápida (ERR), falla para rescatar, ingreso no esperado a terapia intensiva, junto con el reconocimiento de los errores que se producen dentro de un hospital, ha logrado que se enfoquen los esfuerzos en la prevención de las complicaciones durante la estancia intrahospitalaria, obteniendo según el último y más amplio metaanálisis una disminución en el paro cardiorrespiratorio y una reducción en la activación de códigos azules. ${ }^{8}$

Los MEWS representan una necesidad y un parámetro sencillo, no incrementan de manera considerable la carga de trabajo, sino que ofrecen la identificación oportuna de factores que detonan la valoración y manejo por un equipo de médicos experimentados (ERR) y de ser necesario su eventual traslado a terapia intermedia o intensiva, y de esta manera se evita la falla para rescatar, misma que se define como la falta de reconocimiento o respuesta al deterioro del paciente. ${ }^{9}$ Incluso se ha planteado su aplicación de manera rutinaria por parte de enfermería. ${ }^{1}$
Hasta el momento no se ha identificado cuál es el mejor puntaje. Churpek reporta para el SEWS una sensibilidad de $55 \%$, especificidad de $85 \%$; MEWS sensibilidad de $67 \%$, especificidad de $80 \%$; ViEWS sensibilidad de $60 \%$ y especificidad de $83 \%$; y CART sensibilidad de $61 \%$ y especificidad de $84 \%$. Al momento no en todos se ha validado o identificado claramente su utilidad en particular, ${ }^{10}$ pero en general se ha demostrado una disminución en el APACHE II calculado a su ingreso de 23.5 a $16.6 \%$ al aplicarse el MEWS ${ }^{1}$ una reducción en la incidencia de códigos azules hasta en $70 \%$ y un aumento en los llamados del equipo de respuesta rápida en $246 \%,{ }^{11,12}$ además de identificar la mortalidad general y la mortalidad por arresto cardiaco.

Desde 2014 el Hospital Ángeles Pedregal cuenta con un ERR que aplica un MEWS que ha demostrado una disminución en la mortalidad de $37 \%$ comparada con $21 \%$ en los pacientes ingresados en la unidad de terapia intensiva. ${ }^{13,14}$

Debido a que la información reportada del instrumento MEWS no ha sido consistente entre los diferentes autores, sin olvidar que el personal médico lo utiliza a conveniencia con base en los recursos disponibles en sus unidades de atención hospitalaria y sin vigilar la adherencia del mismo, el presente trabajo pretende analizar la eficacia del MEWS validado en comparación con el esquema que actualmente se utiliza en los pacientes en estado perioperatorio con la finalidad de identificar mejoría en las condiciones de atención clínica, reducción de complicaciones y tasa de mortalidad de estos pacientes. ${ }^{15}$

\section{MATERIAL Y MÉTODOS}

Se realizó un ensayo clínico concurrente en el que se incluyeron todos los pacientes hombres y mujeres tratados por servicios no pediátricos, ingresados por urgencia, traslado o cirugía programada que requirieran tratamiento quirúrgico (cirugía general, cirugía oncológica, cirugía colorrectal, cirugía bariátrica, cirugía hepatopancreatobiliar) cuyo seguimiento se dio desde su ingreso hasta su egreso hospitalario.

Los criterios fueron los siguientes:

Inclusión: pacientes con diagnóstico que ameritara tratamiento quirúrgico o pacientes postoperados (cirugía general, oncológica, colorrectal, bariátrica, hepatopancreatobiliar) incluyendo los que cursan RCP III. Pacientes que pasan a piso de hospitalización regular posterior a cursar en terapia intensiva/intermedia durante su postoperatorio inmediato.

Eliminación: pacientes trasladados a otra unidad, pacientes trasladados a terapia intermedia o intensiva posterior a cirugía programada o cirugía de urgencia. 
Las variables que se recabaron fueron: sexo, edad, diagnóstico definitivo, ASA, procedimiento, tiempo quirúrgico, tipo de cirugía (abierta, laparoscópica, robótica), tipo de cirugía (urgencia, electiva), fecha de ingreso, fecha de egreso, cirugía, presencia de complicación, tipo de complicación, duración de la complicación, muerte, voceo de código NEWS o HAP, ingreso a la UTI o UTIM y días de estancia intrahospitalaria.

La valoración se realizó de la siguiente manera:

La toma de signos vitales para obtener el puntaje de los algoritmos fue realizada por enfermería, se registró la frecuencia cardiaca, saturación de oxígeno, temperatura y presión arterial con el dispositivo Welch Allyn Spot vital Signs LXi y con el brazalete No. 11, en caso de pacientes con índice de masa corporal mayor de 40 se utilizó el mango No. 13; la frecuencia respiratoria se tomó con conteo directo bajo cronómetro; la uresis se midió cuantificando la cantidad de orina recolectada en bolsa urinaria o en contenedor desechable entre el peso y el tiempo transcurrido.

Se decidió aplicar de manera aleatorizada exclusivamente el MEWSHAP durante un mes y exclusivamente el MEWS durante un segundo mes contiguo (Tablas 1 y 2).
El equipo de investigadores y colaboradores identificó a los pacientes del universo y se aplicó el algoritmo correspondiente al mes en curso. Para los pacientes que cursaran su estancia intrahospitalaria durante el segundo mes del estudio, se continuó aplicando el algoritmo original hasta su alta.

Para el algoritmo HAP, el equipo investigador se basó en el llamado por la enfermera de guardia, médico de guardia o durante el pase de visita matutino/vespertino/nocturno por el residente de cirugía general (como triple verificación). Se valoró la escala de NEWSHAP; en el caso de cumplir con un puntaje de 1-4 se intervino de acuerdo con criterio y comunicación efectiva con su médico tratante y se revaloró a las cuatro horas, mismo que en caso de mejoría se pasó a vigilancia cada ocho horas, y en caso de deterioro o de continuar en el mismo estado se voceó al ERR.

En caso de obtener de cinco a seis puntos o tres en una misma categoría, se intervino de la misma manera y se revaloró cada 30 minutos, actuando de la misma manera en caso de no mejoría.

Al obtener siete puntos o más o en caso de que impresionara la gravedad, se voceó al ERR.

En caso de que la enfermedad del paciente fuera irreversible o no tuviera deseos de reanimación, las alteraciones fueron notificadas sólo a su médico tratante.

Tabla 1: Variables y puntajes del Modified Early Warning Score Hospital Ángeles Pedregal (MEWSHAP).

\begin{tabular}{|c|c|c|c|c|c|c|c|}
\hline Puntaje & 3 & 2 & 1 & 0 & 1 & 2 & 3 \\
\hline Presión sistólica, mmHg & $<90$ & $91-110$ & $101-110$ & $111-219$ & - & - & $>220$ \\
\hline Frecuencia cardiaca, Ipm & $<40$ & - & $41-50$ & $51-90$ & $91-110$ & $111-130$ & $>131$ \\
\hline Frecuencia respiratoria, rpm & $<8$ & - & $9-11$ & $12-20$ & - & $21-24$ & $>25$ \\
\hline Temperaturam, ${ }^{\circ} \mathrm{C}$ & $<35$ & - & $35.1-36$ & $36.1-38$ & $38.1-39$ & $>39.1$ & - \\
\hline Consciencia & - & - & - & Reactivo & - & - & No reactivo \\
\hline
\end{tabular}

Tabla 2: Modified Early Warning Score Stenhouse (MEWSS).

\begin{tabular}{|c|c|c|c|c|c|c|c|}
\hline Puntaje & 3 & 2 & 1 & 0 & 1 & 2 & 3 \\
\hline Presión sistólica, mmHg & $<70$ & $71-80$ & $81-100$ & 101-199 & - & $>200$ & - \\
\hline Frecuencia cardiaca, Ipm & - & $<40$ & $41-50$ & $51-100$ & $101-110$ & $111-129$ & $>130$ \\
\hline Frecuencia respiratoria, rpm & - & $<9$ & - & $9-14$ & $15-20$ & $21-29$ & $>30$ \\
\hline Temperatura, ${ }^{\circ} \mathrm{C}$ & - & $<35$ & - & $35-38.4$ & - & $>38.5$ & - \\
\hline Consciencia según RASS & - & - & - & Alerta & $\begin{array}{c}\text { Reactivo a } \\
\text { voz }\end{array}$ & $\begin{array}{c}\text { Reactivo a } \\
\text { dolor }\end{array}$ & Sin respuesta \\
\hline & & & & $0 a+3$ & -1 a -3 & -4 & -5 \\
\hline Gasto urinario & Sin gasto & $<1 \mathrm{~mL} / \mathrm{kg} / 2 \mathrm{~h}$ & $<1 \mathrm{~mL} / \mathrm{kg} / \mathrm{h}$ & - & $>3 \mathrm{~mL} / \mathrm{kg} / 2 \mathrm{~h}$ & - & - \\
\hline
\end{tabular}

RASS = escala de la agitación y sedación Richmond (por sus siglas en inglés). 
Figura 1: Algoritmo de actuación de acuerdo con puntaje Modified Early Warning Score Hospital Ángeles Pedregal. $\mathrm{ERR}$ = equipos de respuesta rápida; $\mathrm{UCI}=$ Unidad de Terapia Intensiva.

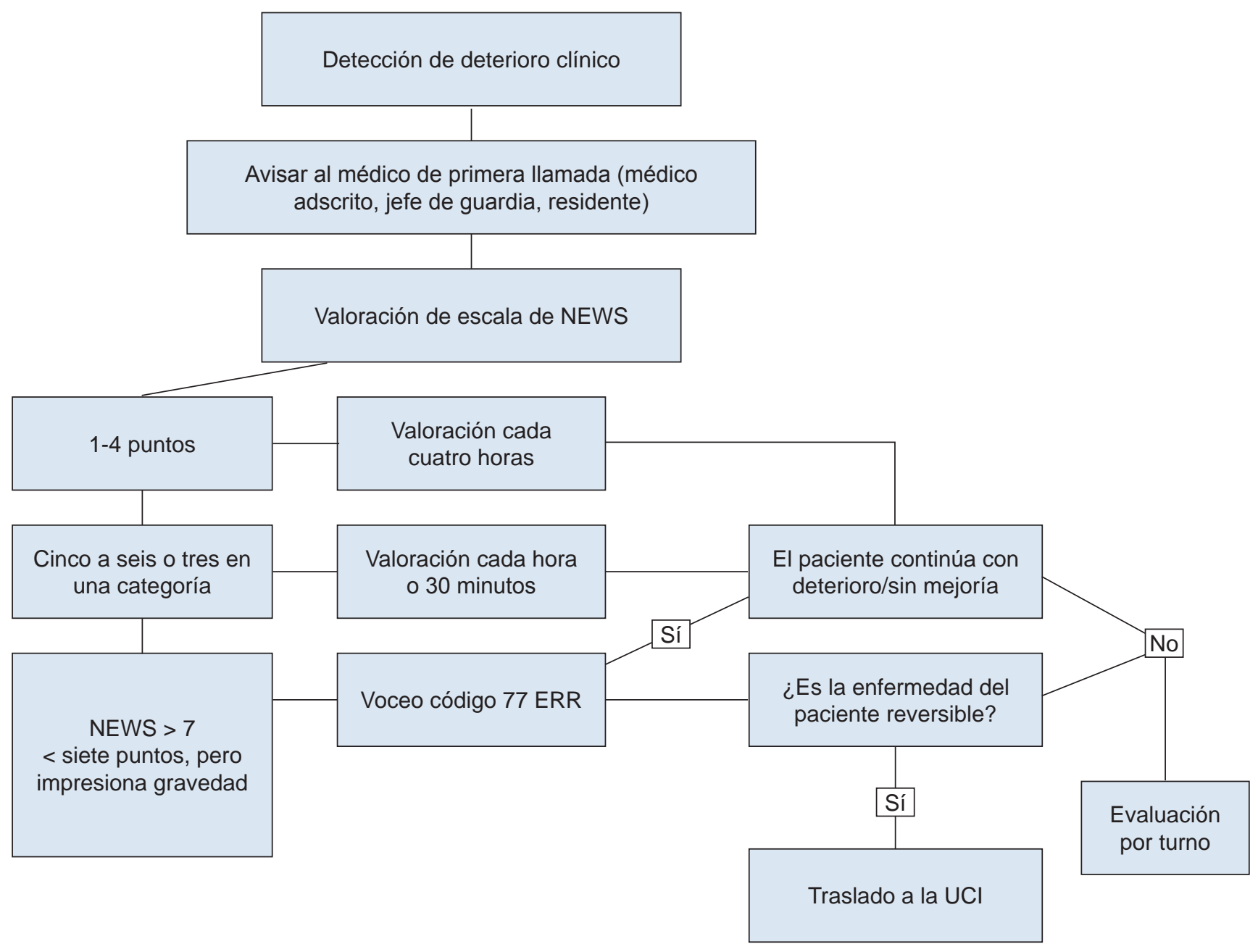

En caso de que la enfermedad del paciente fuera reversible y de acuerdo con valoración del ERR, se trasladó a la UTI o UTIM (Figura 1).

Durante el segundo mes el equipo investigador se basó en el llamado por la enfermera de guardia, médico de guardia o durante el pase de visita matutino/vespertino/ nocturno por el residente de cirugía general (como triple verificación). Se valoró la escala de NEWS; en el caso de cumplir con un puntaje de uno a dos se realizó cuidado habitual, con un puntaje de tres el médico residente de cirugía ajustó manejo y se aumentó el monitoreo clínico. $\mathrm{Al}$ obtener cuatro puntos se reportó a médico tratante, se evaluó el "ABCDE" de reanimación, se inició oxígeno, ajuste de soluciones, se estableció acceso intravenoso y se reevaluó; en caso de mantenerse estático o con deterioro, se voceó al ERR; y en caso de obtener puntaje de cinco puntos o más, se voceó inmediatamente al ERR (Figura 2).

\section{Procesamiento de datos}

La información se recopiló de manera manual en hoja de captación de datos, posteriormente en formato de Microsoft office Excel, donde se codificaron las variables y se vaciaron en base de datos del mismo programa. El análisis estadístico se realizó con $\mathrm{R}$ (versión 3.6.1). Todas las variables numéricas (edad, tiempo quirúrgico, duración de complicación y días de estancia intrahospitalaria $(\mathrm{DEIH})$ se valoraron con medidas de tendencia central y dispersión, para la edad y tiempo de cirugía se realizó t de Student. Para seleccionar el algoritmo con resultados óptimos se integraron los valores de sensibilidad y especificidad en una curva ROC. Se fijó como umbral el puntaje para activar el sistema de respuesta, siete para MEWSHAP y cinco para MEWSS. Por último, se analizaron todas las complicaciones detectadas, para establecer 
la relación entre las complicaciones y la edad se utilizó la $\mathrm{D}$ de Cohen, para la relación entre edad y tiempo de cirugía se realizó una correlación lineal de Pearson y para los días de estancia intrahospitalaria se utilizó media y prueba de los rangos con signo de Wilcoxon.

\section{RESULTADOS}

Se incluyó en el estudio un total de 310 pacientes, de los cuales se eliminaron dos y se excluyeron 13 por cambio de hospital y traslado directo a UTI/UTIM respectivamente, dichos pacientes ingresaron de manera directa de urgencia sin pasar por hospitalización, o que durante su estancia en hospitalización presentaron puntaje de 0 . La muestra final fue de 295 pacientes, de los cuales se obtuvo una media de edad de 45.8 años ( \pm 16.94 ), con una proporción hombre:mujer de 144:151. De estos pacientes, $78.9 \%$ (233/295) fueron procedimientos electivos y $21.02 \%$ (62/295) procedimientos de urgencia. Las tres cirugías más frecuentes fueron colecistectomías (24.4\%), funduplicaturas $(13.56 \%)$ y plastias inguinales (10.85\%). De los procedimientos realizados, 60.34\% fue de tipo laparoscópico y $36.9 \%$ abierto; el promedio de tiempo de cirugía fue de 70 minutos $( \pm 60.07)$. Por último, la mayoría de los pacientes obtuvieron un ASA entre I y II (Tabla 3).

Figura 2: Algoritmo de actuación Modified Early Warning Score Stenhouse.

$E R R=$ equipos de respuesta rápida; IV = intravenoso.

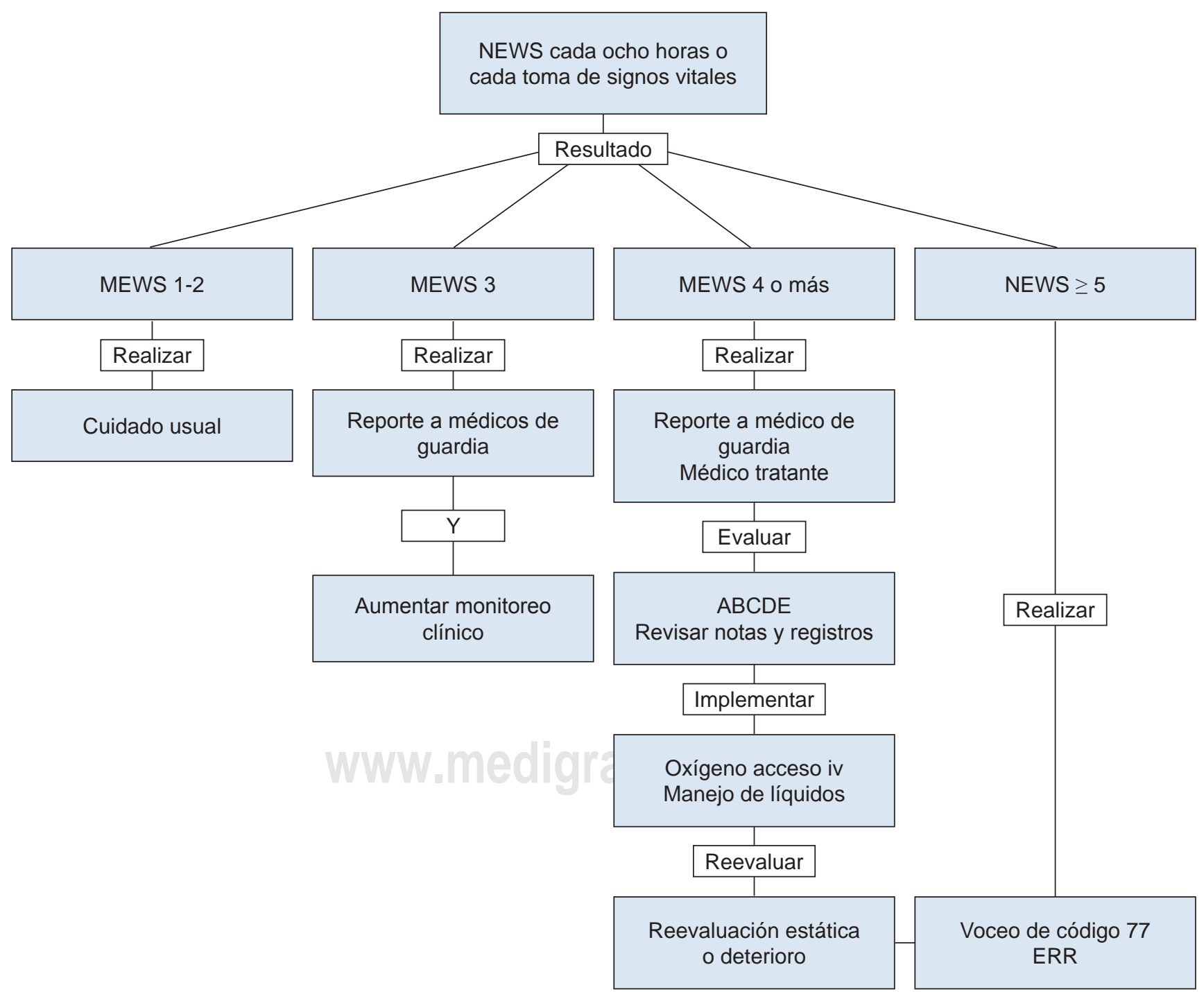


Tabla 3: Resultados generales.

\begin{tabular}{|c|c|c|c|}
\hline & $\begin{array}{c}\text { Total } \\
(\mathrm{N}=295) \\
\mathrm{n}(\%)\end{array}$ & $\begin{array}{c}\text { HAP } \\
(n=163) \\
n(\%)\end{array}$ & $\begin{array}{c}\text { MEWS } \\
(n=132) \\
n(\%)\end{array}$ \\
\hline \multicolumn{4}{|l|}{ Género } \\
\hline $\begin{array}{l}\text { Proporción género } \\
(\mathrm{M}: \mathrm{F})\end{array}$ & 144:151 & $83: 80$ & $61: 71$ \\
\hline \multicolumn{4}{|l|}{ Edad (años) } \\
\hline Promedio & 45.88 & 48.04 & 43.23 \\
\hline Máx. & 94 & 94 & 81 \\
\hline Mín. & 14 & 14 & 15 \\
\hline $\mathrm{DE}( \pm)$ & 16.94 & 17.33 & 16.13 \\
\hline \multicolumn{4}{|l|}{ Qx } \\
\hline \multicolumn{4}{|c|}{ Procedimiento (10 más frecuentes) } \\
\hline Colecistectomía & $72(24.41)$ & $43(33.33)$ & $29(28.16)$ \\
\hline Funduplicatura & $40(13.56)$ & $26(20.16)$ & $14(13.59)$ \\
\hline Plastia inguinal & $32(10.85)$ & $15(11.63)$ & $17(16.50)$ \\
\hline Apendicectomía & $25(8.47)$ & $13(10.08)$ & $12(11.65)$ \\
\hline Hemorroidectomía & $20(6.78)$ & $8(6.20)$ & $12(11.65)$ \\
\hline Laparotomía & $11(3.73)$ & $4(3.10)$ & $7(6.80)$ \\
\hline Tiroidectomía total & $10(3.39)$ & $7(5.43)$ & $3(2.91)$ \\
\hline $\begin{array}{l}\text { Drenaje de } \\
\text { absceso }\end{array}$ & $8(2.71)$ & $6(4.65)$ & $2(1.94)$ \\
\hline Bypass gástrico & $7(2.37)$ & $1(0.78)$ & $6(5.83)$ \\
\hline Fistulectomía & $7(2.37)$ & $6(4.65)$ & $1(0.97)$ \\
\hline \multicolumn{4}{|l|}{ Tipo } \\
\hline Abierta & 109 (36.95) & $58(35.58)$ & $51(38.64)$ \\
\hline Laparoscópica & $178(60.34)$ & $100(61.35)$ & 78 (59.09) \\
\hline Robótica & $7(2.37)$ & $4(2.45)$ & $3(2.27)$ \\
\hline Conversión & $1(0.34)$ & $1(0.61)$ & $0(0)$ \\
\hline Electiva & $233(78.98)$ & $124(76.07)$ & $109(82.58)$ \\
\hline Urgencia & $62(21.02)$ & $39(23.93)$ & $23(17.42)$ \\
\hline \multicolumn{4}{|l|}{ Tiempo cirugía } \\
\hline Mediana & 70 & 60 & 89 \\
\hline Máx. & 480 & 450 & 480 \\
\hline Mín. & 25 & 25 & 30 \\
\hline $\mathrm{DE}( \pm)$ & 60.07 & 62.57 & 56.95 \\
\hline \multicolumn{4}{|l|}{ Complicaciones } \\
\hline $\begin{array}{l}\text { Número de } \\
\text { complicaciones }\end{array}$ & 21 & 15 & 6 \\
\hline Ingreso UTI/UTIM & 6 & 3 & 3 \\
\hline \multicolumn{4}{|l|}{ ASA } \\
\hline I & $161(55.14)$ & $82(50.62)$ & $79(60.77)$ \\
\hline II & $119(40.75)$ & $75(46.30)$ & $44(33.85)$ \\
\hline III & $11(3.77)$ & $4(2.47)$ & $7(5.38)$ \\
\hline IV & $1(0.34)$ & $1(0.62)$ & $0(0.00)$ \\
\hline
\end{tabular}

HAP = Hospital ángeles Pedregal; MEWS = Modified Early Warning Score; M:F = masculino:femenino; $\mathrm{DE}=$ desviación estándar; $\mathrm{Qx}=$ cirugía; UTI/UTIM = Unidad de Terapia Intensiva/Unidad de Terapia Intensiva Móvil.

\section{Algoritmo HAP vs. algoritmo MEWS}

Para comparar los sistemas de alarma durante el periodo del algoritmo MEWSHAP (primer periodo) se registraron 163 pacientes, mientras que durante el periodo MEWSS (segundo periodo) se registraron 132 pacientes. Las variables analizadas (edad t de Student $p=0.015$, tiempo de cirugía t de Student $p=0.55$ ) no mostraron diferencias estadísticamente significativas entre los dos grupos.

De las 21 complicaciones, 15 surgieron durante el periodo MEWSHAP y seis durante el periodo MEWSS. De las 15 complicaciones durante el MEWSHAP, tres ingresaron a UTI/UTIM posterior a ingreso por urgencias, dos colecistectomías y una laparotomía. Un paciente presentó taquicardia paroxística supraventricular, tuvo MEWSHAP de tres puntos, sin ameritar voceo, pero aun así se hizo el ingreso a UTIM con resolución de su complicación en 30 minutos. El segundo paciente cursó con taquicardia, oliguria e hipotensión por dos horas, con MEWSHAP de siete, se realizó voceo ingresando a UTIM, posteriormente ameritó reoperación identificando una hernia interna. La tercera paciente con 72 horas de hipotensión, oliguria, taquicardia MEWSHAP de siete, con voceo durante su estancia en piso y reintervención quirúrgica en una ocasión y con segunda reoperación falleció posteriormente en la UTI.

Para el periodo MEWSS también tres pacientes ingresaron a la UTI/UTIM por procedimientos quirúrgicos de urgencia, dos anastomosis intestinales (oclusión intestinal y sangrado diverticular incoercible) y una apendicectomía (apendicitis complicada fase IV). La primera dehiscencia posterior a oclusión tuvo lugar 15 horas postoperatorias y alcanzó MEWSS de dos, sin voceo, ameritando reoperación e ingreso a UTIM postoperatorio. La dehiscencia del muñón apendicular cursó 10 horas postoperatorias, alcanzó MEWSS de dos, sin voceo, ameritando reoperación e ingreso a la UTIM postoperatorio. El tercer ingreso del sangrado diverticular y resección colónica cursó con dos horas de hipotensión, taquicardia y oliguria con MEWSS de cinco e ingresó a la UTI mejorando al manejo médico (Figura 3).

Para comparar la sensibilidad de ambos puntajes se fijó como umbral el puntaje para activar el sistema de respuesta, siete para MEWSHAP y cinco para MEWSS, y el número de ingresos a la unidad de cuidados intensivos/ intermedios por la activación del mismo. Para MEWSHAP se obtuvo una sensibilidad de $66 \%$, mientras que para MEWSS fue de 33\%.

Se integraron los valores de sensibilidad y especificidad en una curva ROC para determinar el área bajo la curva, primero tomando en cuenta como valor verdadero positivo cuando se activó el sistema de alarma y el paciente ingresó 
a la UTI/UTIM (Figura 4); el algoritmo MEWSS área under the curve (AUC $=0.75$ ) demostró ser más eficaz que el algoritmo MEWSHAP (AUC [area under the curve] $=0.59$ ), aunque ninguno de los dos alcanzó un área bajo la curva $>0.9$, lo que indicaría un buen predictor (Figura 5).

\section{Horas de duración de complicación}

Las 15 complicaciones presentes durante el periodo MEWSHAP tuvieron una duración promedio de 11.5 horas, aunque durante el periodo MEWSS tuvieron una duración promedio de 9.83 horas, sin mostrar una relación significativa entre los algoritmos y la duración de las complicaciones como se muestra en la Figura 6 con $p=0.72$.

\section{Días de estancia intrahospitalaria}

La estancia intrahospitalaria promedio fue de 2.6 DEIH, aunque para los pacientes que presentaron alguna complicación durante el periodo MEWSHAP la estancia aumentó a 6.86 días, y durante el periodo MEWSS se prolongó hasta 10.5 días, lo que se evidencia en la Figura 7 con $\mathrm{p}=7.3 \mathrm{e}-08$.

\section{Complicaciones en relación con la edad}

De las variables con una asociación más clara se encuentra la de la presencia de complicaciones con la edad, donde a mayor edad es más probable que surjan complicaciones, la cual se comprobó mediante el método de $\mathrm{D}$ de Cohen con un efecto amplio ( $\mathrm{D}$ de Cohen $=-1.05$ ) con intervalo de confianza de $95 \%$ (-1.50 a -0.59) como puede observarse claramente en la Figura 8.

Figura 3: Presencia de complicaciones, ingreso a la UTI/UTIM y voceo de código por algoritmo.



Figura 4: Curva ROC con la activación del sistema de respuesta, considerando sólo la sensibilidad con el puntaje para ingreso a la UTI/UTIM. A) Algoritmo HAP (MEWSHAP) y B) algoritmo NEWS (MEWSS).


Figura 5: Curva ROC con la valoración de ambos algoritmos para la detección de complicaciones. A) Algoritmo HAP (MEWSHAP) y B) algoritmo NEWS (MEWSS).

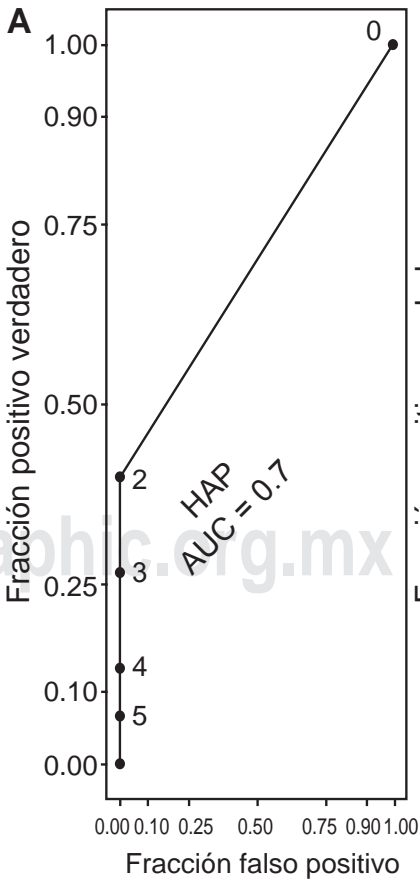


Figura 6: Prueba de los rangos con signo de Wilcoxon relacionando ambos algoritmos con la duración de las complicaciones.



\section{Edad y tiempo de cirugía}

Para la correlación de la edad y el tiempo quirúrgico, por tratarse de dos variables numéricas, se hizo una correlación lineal de Pearson, en la que se puede observar por la distribución de las muestras que no existe correlación entre el tiempo de cirugía y las complicaciones $(R=0.14)$ (Figura 9).

\section{Complicaciones menores}

Las complicaciones que no ameritaron voceo ni traslado a la UTI/UTIM y que surgen más en el periodo MEWSHAP fueron oliguria en 10 casos, cumpliendo con criterio de lesión renal aguda AKIN 1, seguida de hipotensión en tres casos. Durante el periodo MEWSS se presentó oliguria en dos casos, seguidos de un caso de hipotensión.

\section{DISCUSIÓN}

La subvaloración y apego exclusivo al juicio clínico por parte de enfermeras y médicos puede producir un deterioro por omisión en la salud de los pacientes hospitalizados y llevarlos a presentar complicaciones mediatas o tardías en distintos aparatos y sistemas. No sabemos si obtenemos el resultado óptimo al aplicar el protocolo establecido en nuestro hospital; aunque ya contamos con resultados, vale la pena discernir entre puntajes para mejorar aún más los resultados en beneficio del paciente. ${ }^{14}$

El protocolo del hospital se planteó para realizarse en meses contiguos, pues en la actualidad existe un algoritmo para el equipo de respuesta rápida que es difícil modificar si no se obtienen resultados totalmente contrarios, hecho

Figura 7: Prueba de los rangos con signo de Wilcoxon relacionando DEIH con presencia o ausencia de complicaciones.

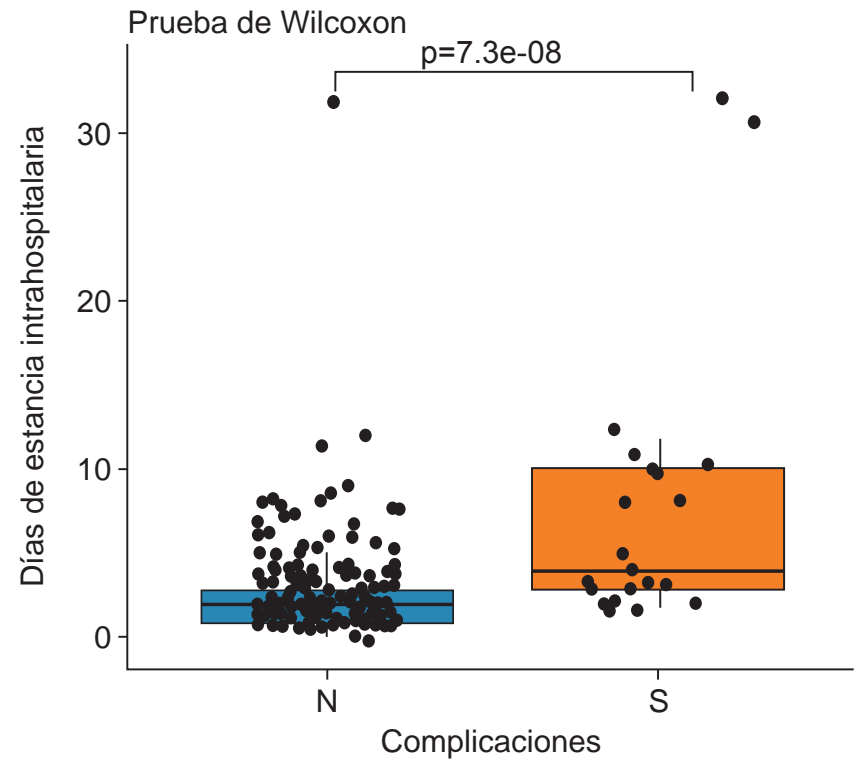

Figura 8: La presencia de complicaciones de acuerdo con la edad.

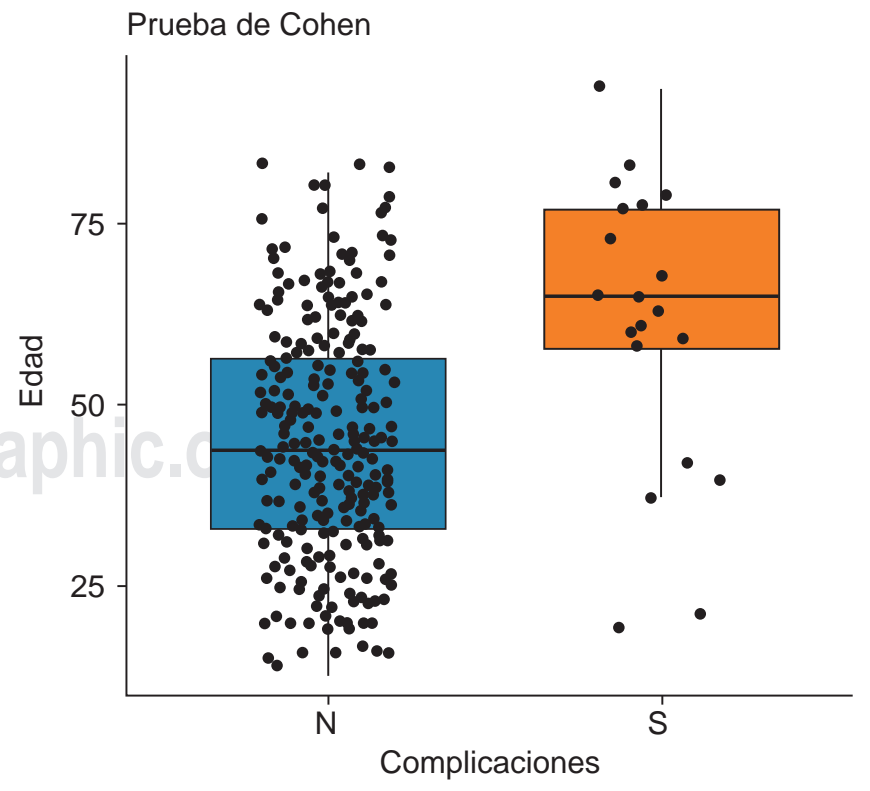


Figura 9: Distribución lineal del tiempo de cirugía de acuerdo con la edad.

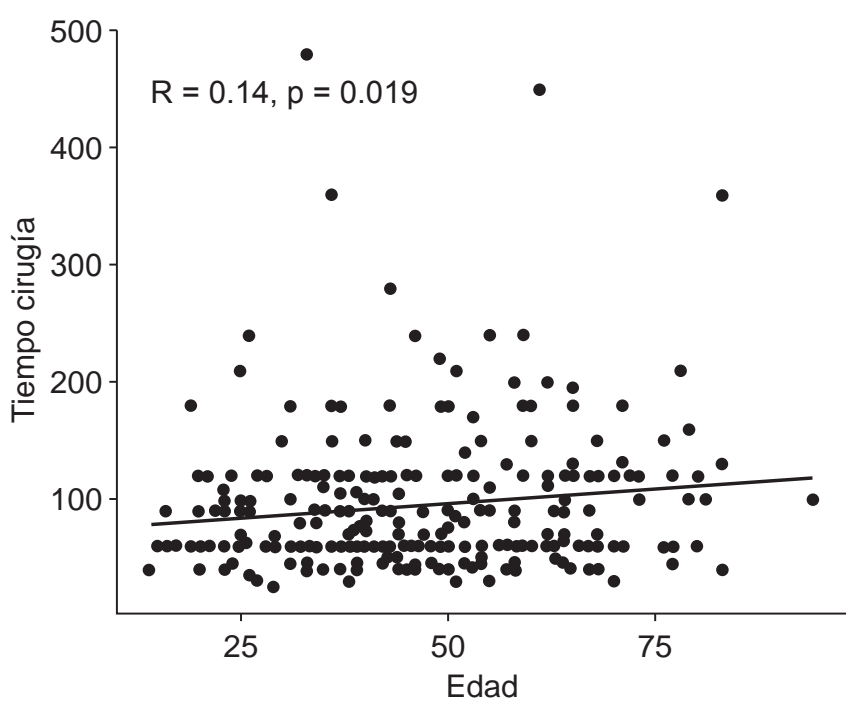

por el cual la muestra no pudo ser más grande y se consideró aproximada a las ya existentes en estudios como los de Gardner Thorpe. Además, cada protocolo activa un protocolo de actuación diferente, por lo que no es posible comparar los dos en el mismo paciente.

El enfoque de dichos algoritmos se da con base en la observación previa de la subestimación de las complicaciones quirúrgicas por parte del algoritmo vigente en el hospital, motivo por el cual consideramos necesario compararlo contra uno previamente validado.

Es importante notar que la mayoría de cirugías fueron programadas, casi $80 \%$, de las cuales $95 \%$ se practicaron en pacientes ASA I-II, por lo que es imperativo tener un buen algoritmo de vigilancia perioperatoria y disminuir la mortalidad, dado que la posibilidad de complicaciones graves o muerte es baja en el rango de hasta $0.4 \%$ en ASA II. Tuvimos una mortalidad de $0.3 \%$, por lo que con una muestra semejante a estudios previos obtuvimos la misma mortalidad respecto al ASA.

Las admisiones no planeadas a terapia intensiva rondan de $0.5-1 \%$, obtuvimos mayor admisión no planeada a pesar de contar con un algoritmo vigente, ubicándonos en $2.03 \% .{ }^{1}$ Los voceos sólo fueron dos, siendo menores incluso que los ingresos a cuidados especiales, esto con base en que el día de hoy existe un sistema que funciona. La diferencia aumentó a $243 \%$ en hospitales sin algoritmos funcionales. ${ }^{11,12}$

Aun cuando nuestro hospital es eminentemente laparoscópico y en efecto la mayoría de cirugías se realizaron de manera laparoscópica, $60 \%$ vs. $36 \%$ abiertas, un porcentaje sorprendente, probablemente por la relación con la incidencia de cáncer que tenemos en el hospital. Cuando revisamos los datos de nuestra población no existió diferencia estadísticamente significativa entre las variables generales del estudio, por lo que no hay diferencias que provoquen un sesgo en la comparación.

Aunque los objetivos terminales de dichos algoritmos son evitar el deterioro y el ingreso a la unidad de cuidados especiales, es cierto que en caso de necesitarlo, debe ser de manera oportuna. Gardner Thorpe en 334 pacientes quirúrgicos obtuvo una sensibilidad y especificidad de 75 y $83 \%$ respectivamente, ${ }^{3}$ siendo menor en nuestro caso MEWSHAP $66 \%$ vs. $33 \%$ de MEWSS. El área bajo la curva mostró un mejor resultado de MEWSS (0.75) vs. MEWSHAP (0.59). ${ }^{3}$ Cuando integramos las curvas ROC con todos los valores obtenidos de la valoración de ambos algoritmos y tanto las complicaciones menores como los ingresos a la UTI/UTIM, el algoritmo MEWSS (AUC $=0.91$ ) sigue siendo más eficaz en comparación con el MEWSHAP (AUC = 0.7) para la detección de cualquier tipo de complicación, alcanzando un $\mathrm{A} \cup \mathrm{C}>0.9$.

Cabe resaltar que de los seis ingresos a cuidados especiales, sólo uno fue por causa cardiológica pura (arritmia) y que aun así el algoritmo no lo detectó para ingresarlo en cuidados especiales, área a la que entró por protocolo previo del hospital para la cardioversión farmacológica. Otro paciente fue ingresado por anemia y mejoró al manejo médico endoscópico. Los siguientes tres pacientes ameritaron manejo quirúrgico. Se obtuvieron resultados mixtos, pues la sensibilidad para el ingreso a cuidados intensivos es mejor en el algoritmo MEWSHAP, pero si lo comparamos con la detección de todas las complicaciones, el algoritmo MEWSS da mejores resultados. Lo anterior se debate con Hollis, quien observó que en pacientes quirúrgicos con mayor puntaje se previenen complicaciones más graves, hecho que en nuestra muestra no se corroboró, pues los pacientes que ameritaron reoperación no activaron el código sin tener una causa evidente del origen del mismo. ${ }^{6}$

Se observó además que a pesar de la mayor duración de complicaciones durante el periodo MEWSHAP, no se encontró significancia estadística, condición que tal vez para el presente trabajo no es beneficiosa, pero abre la posibilidad de seguir investigando el mejor puntaje para nuestra población sin someterla a riesgo de mayor duración de complicaciones o no identificación de las mismas. Albur observa que el hecho de mejorar el MEWS a las 48 horas es de buen pronóstico, situación que no estudiamos, pero sentamos precedentes para evaluar posteriormente el ingreso a cuidados especiales.

Los días de estancia intrahospitalaria (DEIH) se prolongaron de 6.86 días durante el periodo MEWSHAP vs. 10.5 días durante el periodo MEWSS. Desafortunadamente, la muestra es insuficiente para tener poder estadístico y saber 
si es por azar que durante el periodo MEWSS los pacientes se quedan más tiempo, lo anterior probablemente en relación con la presencia de dos fugas de anastomosis durante este último periodo que ameritaron más de 30 DEIH, mismas que a pesar de presentar una complicación quirúrgicamente grave, de manera paradójica no accionaron el algoritmo MEWSS.

Como era de esperarse la edad fue un factor que se asoció a la aparición de complicaciones, hecho reportado previamente, a diferencia del tiempo quirúrgico, condición normalmente estigmatizada durante la cirugía, pero que no mostró significancia estadística en el desarrollo de complicaciones posteriores.

Especial mención merece el caso de defunción durante el periodo HAP, que aunque fue detectado a tiempo y voceado, resultó irrecuperable a pesar del máximo esfuerzo; importantes también los dos casos durante el periodo MEWS que ameritaron reoperación sin requerir voceo, que si bien no tienen significancia estadística, pueden insinuar la baja especificidad que se obtendría de manera conjugada para los casos quirúrgicos específicamente; calculamos sensibilidad de $71 \%$ y especificidad de $46 \%$.

Las conclusiones particulares para nuestro hospital hacen pensar que el criterio clínico tiene aún mucho que ofrecer a los pacientes puramente quirúrgicos y que si bien los EWS tienen un papel y buenos resultados en la población general, podríamos considerar ajustar un EWS para pacientes quirúrgicos específicamente. Smith reporta que dadas las limitaciones del diseño de los estudios, el impacto no ha sido correctamente estudiado o medido. ${ }^{16}$ La última actualización llamada NEWS 2 salió en diciembre de 2017 mientras se realizaba este estudio, por lo que valdría la pena comparar el presente algoritmo con la última versión.

\section{CONCLUSIONES}

Con una muestra semejante a estudios previos y sin diferencia estadística en los datos generales de la muestra obtuvimos la misma mortalidad respecto al ASA. Observamos que la edad se correlaciona con la presencia de complicaciones y que el tiempo de cirugía no tiene relación. Obtuvimos mayor admisión no planeada a pesar de contar con un algoritmo vigente. Demostramos mejor sensibilidad del algoritmo MEWSHAP y mejor especificidad del algoritmo MEWSS; aunque enfocados en las complicaciones que ameritaron reoperación, ninguno de los dos activó el código y a pesar de que observamos mayor duración de complicaciones durante el periodo NEWSHAP, no tuvo significancia estadística, incluso si tuvimos mayores DEIH durante el algoritmo MEWSS. Por lo anterior consideramos que el algoritmo actualmente vigente en nuestro hospital es apto para la aplicación en pacientes quirúrgicos, aun cuando su aplicación no reemplaza la revisión clínica en cuanto a complicaciones tempranas que ameritan reoperación, pero abre la puerta para ajustes específicos en nuestra población.

\section{REFERENCIAS}

1. Stenhouse C, Coates S, Tivey M, Allsop P, Parker T. Prospective evaluation of a modified early warning score to aid earlier detection of patients developing critical illness on a general surgical ward. $\mathrm{Br}$ J Anaesth. 2000; 84 (5): 663.

2. Ludikhuize J, Smorenburg SM, de Rooij SE, de Jonge E. Identification of deteriorating patients on general wards; measurement of vital parameters and potential effectiveness of the Modified Early Warning Score. J Crit Care. 2012; 27 (4): 424.e7-13.

3. Gardner-Thorpe J, Love N, Wrightson J, Walsh S, Keeling N. The value of Modified Early Warning Score (MEWS) in surgical in-patients: a prospective observational study. Ann R Coll Surg Engl. 2006; 88 (6): 571-575.

4. Jansen JO, Cuthbertson BH. Detecting critical illness outside the ICU: the role of track and trigger systems. Curr Opin Crit Care. 2010; 16 (3): 184-190.

5. Smith GB, Prytherch DR, Schmidt PE, Featherstone PI. Review and performance evaluation of aggregate weighted 'track and trigger' systems. Resuscitation. 2008; 77 (2): 170-179.

6. Hollis RH, Graham LA, Lazenby JP, Brown DM, Taylor BB, Heslin MJ et al. A role for the early warning score in early identification of critical postoperative complications. Ann Surg. 2016; 263 (5): 918-923.

7. Centre for Clinical Practice at NICE (UK). Acutely ill patients in hospital: recognition of and response to acute illness in adults in hospital [Internet]. London: National Institute for Health and Clinical Excellence (UK); 2007.

8. Maharaj R, Raffaele I, Wendon J. Rapid response systems: a systematic review and meta-analysis. Crit Care. 2015; 19 (1): 254.

9. Morgan RJ, Wright MM. In defence of early warning scores. $\mathrm{Br}$ J Anaesth. 2007; 99 (5): 747-748.

10. Gao H, McDonnell A, Harrison DA, Moore T, Adam S, Daly K et al. Systematic review and evaluation of physiological track and trigger warning systems for identifying at-risk patients on the ward. Intensive Care Med. 2007; 33 (4): 667-679.

11. Fullerton JN, Price CL, Silvey NE, Brace SJ, Perkins GD. Is the Modified Early Warning Score (MEWS) superior to clinician judgement in detecting critical illness in the pre-hospital environment? Resuscitation. 2012; 83 (5): 557-562.

12. Maupin JM, Roth DJ, Krapes JM. Use of the Modified Early Warning Score decreases code blue events. It Comm J Qual Patient Saf. 2009; 35 (12): 598-603.

13. Elguea EPA, Hernández CC, Ramírez RL, García GN, López LC, Gómez GB et al. Efectividad del equipo de respuesta rápida en el Hospital Ángeles Pedregal. Acta Med. 2017; 15 (3): 181-188.

14. Hernández AJF, Villanueva SE, Cortés RJJP, Cabrera JR, Martínez LAC, Castillo GFA et al. Sistemas de advertencia temprana por puntaje. Early Warning Scores (EWS). Acta Med. 2019; 17 (3): 252-258.

15. Churpek MM, Yuen TC, Edelson DP. Risk stratification of hospitalized patients on the wards. Chest. 2013; 143 (6): 17581765.

16. Smith ME, Chiovaro JC, $\mathrm{O}^{\prime}$ Neil M, Kansagara D, Quiñones AR, Freeman $\mathrm{M}$ et al. Early warning system scores for clinical deterioration in hospitalized patients: a systematic review. Ann Am Thorac Soc. 2014; 11 (9): 1454-1465. 